\title{
Rapid Identification of Staphylococcus Species Isolated from Food Samples by Matrix-Assisted Laser Desorption/Ionization Time-of-Flight Mass Spectrometry
}

\author{
Eiseul Kim ${ }^{1 \dagger}$, Hyun-Joong Kim ${ }^{1 \dagger}$, Seung-Min Yang ${ }^{1}$, Chang-Gyeom Kim², Dong-Won $\mathrm{Choo}^{2}$, and Hae-Yeong Kim ${ }^{1 *}$ \\ ${ }^{1}$ Institute of Life Sciences and Resources and Department of Food Science and Biotechnology, Kyung Hee University, Yongin 17104, Republic \\ of Korea \\ ${ }^{2}$ Department of Bioinformatics and Biosystems, Korea Polytechnics, Seongnam 13122, Republic of Korea
}

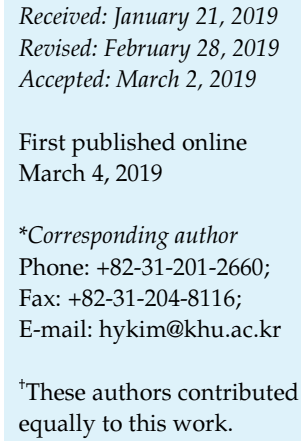

Staphylococcus species have a ubiquitous habitat in a wide range of foods, thus the ability to identify staphylococci at the species level is critical in the food industry. In this study, we performed rapid identification of Staphylococcus species using Matrix-Assisted Laser Desorption/Ionization Time-of-Flight mass spectrometry (MALDI-TOF MS). MALDI-TOF MS was evaluated for the identification of Staphylococcus reference strains $(n=19)$ and isolates ( $n=96)$ from various foods with consideration for the impact of sample preparation methods and incubation period. Additionally, the spectra of isolated Staphylococcus strains were analyzed using principal component analysis (PCA) and a main spectra profile (MSP)-based dendrogram. MALDI-TOF MS accurately identified Staphylococcus reference strains and isolated strains: the highest performance was by the EX method (83.3 89.5\% accuracy) at species level identification (EDT, 70.3 78.9\% accuracy; DT, less than 46.3 63.2\% accuracy) of 24-h cultured colonies. Identification results at the genus level were $100 \%$ accurate at EDT, EX sample preparation and 24-h incubation time. On the other hand, the DT method showed relatively low identification accuracy in all extraction methods and incubation times. The analyzed spectra and MSP-based dendrogram showed that the isolated Staphylococcus strains were characterized at the species level. The performance analysis of MALDI-TOF MS shows the method has the potential ability to discriminate between Staphylococcus species from foods in Korea. This study provides valuable information that MALDI-TOF MS can be applied to monitor microbial populations and pathogenic bacteria in the food industry thereby contributing to food safety.

Keywords: MALDI-TOF MS, Staphylococcus, jeotgal, fermented food, identification, extraction method

\section{Introduction}

The Staphylococcus genus consists of 47 species. Staphylococcus aureus, a species representative of coagulase-positive staphylococci (CoPS), regarded as the pathogenic Staphylococcus, has been a major foodborne pathogen among the many species [1]. However, coagulase-negative staphylococci (CoNS) have recently emerged as major nosocomial pathogens including S. epidermidis, S. haemolyticus, S. saprophyticus, and other species $[2,3]$. Despite some benefits of certain Staphylococcus species, including their use as starter strains for flavor enrichment in dairy-fermented foods and for their positive effects on food quality in the fermentation process of cheese and sausage, researchers have been compelled to pay attention to the pathogenic potential and safety assessment of CoNS by the recent increase in nosocomial infection cases involving CoNS [1, 3, 4]. Due to the ubiquitous habitat of CoNS in a wide range of foods as 
well as in niches of the human body and living areas [2,5], the surveillance and accurate diagnostics regarding CoNS at the species level within the Staphylococcus genus have become critical in the food industry.

For the identification of Staphylococcus species, mannitol salt agar (MSA) has been used and developed for the presumptive isolation of $S$. aureus and staphylococci, however, it has recently been reported that MSA is not sufficient to distinguish between S. aureus and CoNS [6]. In addition, the above technique is time-consuming, laborious, and requires highly trained biologists to conduct. So, additional diagnostic means are needed to accurately distinguish between S. aureus and CoNS at the Staphylococcus species level. To overcome the drawbacks of the phenotypic method, genotypic methods that can be analyzed rapidly and accurately have been used. Among them, sequence analysis of the 16S rRNA gene, a highly conserved region present in bacteria, is most commonly used to identify bacteria [7]. However, the $16 \mathrm{~S}$ rRNA genes of Staphylococcus are closely related to each other and therefore not sufficiently different to discriminate between species [7-9].

Recently, MALDI-TOF MS has emerged as a breakthrough means for the rapid and routine identification of microorganisms with regard to cost-effectiveness, time-savings, high reproducibility, and high reliability [10-12]. MALDITOF MS technology depends on the generation of unique mass spectra captured from a small amount of microbial colony followed by comparison to a reference database containing known microbial spectra for identification of microorganisms [10, 13-15]. Studies on clinical applications for pathogenic staphylococci diagnostics and on the identification of a variety of Staphylococcus species using commercial MALDI-TOF MS systems have been evaluated or compared with other diagnostics [6, 16]. The commercially available Bruker MALDI system is supported by a 38 Staphylococcus species database (Bruker database version 4.0.0.1). In addition to the database, sample-processing methods and growth conditions of bacterial culture can impact the biomass generation and analysis of the subsequent mass spectrum [17, 18]. These effects may lead to false microbiological identification results, and thus a standard protocol for sample-processing according to genus is required.

In this present study, we evaluated the ability of MALDITOF MS to identify Staphylococcus species isolated from food samples. Also evaluated were three extraction methods at various incubation periods for comparison with Staphylococcus reference strains and food-isolated strains to establish an optimal methodology for the identification of Staphylococcus species in the food industry.

\section{Materials and Methods}

\section{Bacterial Strains}

The Staphylococcus species used in this study included 19 reference strains shown in Table 1 . The reference strains were

Table 1. Staphylococcus reference strains used in this study.

\begin{tabular}{lll}
\hline \multicolumn{1}{c}{ Bacterial strains } & \multicolumn{1}{c}{ Strain designations or origins $^{\mathrm{a}}$} & Cluster group $^{\mathrm{b}}$ \\
\hline S. aureus & ATCC 6538, ATCC 6538P, ATCC 29737, NCCP 14560 & S. aureus \\
S. epidermidis & ATCC 12228, ATCC 14990, NCCP 14723 & S. epidermidis \\
S. capitis & NCCP 14663 & S. epidermidis \\
S. caprae & KCTC 3583 & S. epidermidis \\
S. haemolyticus & ATCC 29970 & S. haemolyticus \\
S. hominis & NCCP 10748 & S. haemolyticus \\
S. schleiferi subsp. coagulans & KCCM 41634 & S. hyicus-intermedius \\
S. lugdunensis & NCCP 15630 & S. lugdunensis \\
S. saprophyticus & NCCP 14670 & S. saprophyticus \\
S. xylosus & NCCP 10937 & S. saprophyticus \\
S. lentus & KCCM 41469 & S. sciuri \\
S. sciuri subsp. sciuri & KCCM 41468 & S. sciuri \\
S. warneri & KCTC 3340 & S. warneri \\
S. pettenkoferi & DSM 19554 & - \\
\hline
\end{tabular}

${ }^{a}$ ATCC, American Type Culture Collection; NCCP, National Culture Collection for Pathogens of Korea; KCTC, Korean Collection for Type Culture; KCCM, Korean Culture Center of Microorganisms; DSM, Leibniz Institute DSMZ-German Collection of Microorganisms and Cell Cultures.

${ }^{\mathrm{b}}$ Cluster groups of Staphylococcus species were described from the phylogenetic study based on 16S rRNA gene sequence analysis (Takahashi et al. 1999). 
obtained from the American Type Culture Collection (ATCC, USA), the National Culture Collection for Pathogens (NCCP) in Korea, the Korean Collection for Type Culture (KCTC), the Korean Culture Center of Microorganisms (KCCM) and the Leibniz Institute DSMZ-German Collection of Microorganisms and Cell Cultures (DSMZ). To prepare cells for MALDI-TOF MS analysis, the 19 reference strains were streaked on nutrient agar (BD, USA) and incubated at $37^{\circ} \mathrm{C}$ for $24 \mathrm{~h}$.

\section{Isolation of Staphylococcus Species}

Presumptive Staphylococcus strains were isolated from food products including jeotgal, salted Chinese cabbage, and raw milk by a method modified from previous studies $[5,19]$. Briefly, a $25 \mathrm{~g}$ food sample was added to $225 \mathrm{ml}$ of buffered peptone water (BD) in stomacher filter bags (Seward Limited, United Kingdom), and the mixture was homogenized at $230 \mathrm{rpm}$ for $1.5 \mathrm{~min}$ by a stomacher (Stomacher 400 Circulator, Seward Limited, United Kingdom). The homogenized samples were serially diluted in buffered peptone water and spread onto Mannitol Salt Agar plates (BD). The plates were then incubated at $37^{\circ} \mathrm{C}$ for $24 \sim 48 \mathrm{~h}$ and the colonies of presumptive Staphylococcus (yellow- or redcolored colonies of $2 \sim 5 \mathrm{~mm}$ size) were isolated by their morphological features on Mannitol Salt Agar medium.

\section{Sample Preparation for MALDI-TOF MS}

A loopful of each Staphylococcus strain was streaked on a nutrient agar (NA) (BD) plate and incubated at $37^{\circ} \mathrm{C}$. Each strain was sampled at $24 \mathrm{~h}$ (Day-1), $48 \mathrm{~h}$ (Day-2), and $72 \mathrm{~h}$ (Day-3). The sampled bacterial mass was prepared for MALDI-TOF MS analysis by three different sample preparation methods recommended by the manufacturer (Bruker Daltonics, Germany) and referenced by previously reported protocols [20, 21]; direct transfer (DT), extended direct transfer (EDT), and full formic acid extraction (EX). Expendable supplies used were MSP 96 target plates (Bruker Daltonics), $\alpha$-acyano-4-hydroxycinnamic acid (HCCA) matrix solution in acetonitrile/water/trifluoroacetic acid (TFA) (50:47.5:2.5 [v/v]), and 70\% formic acid.

For the direct transfer method (DT); a single colony was deposited directly on an assigned position of an MSP 96 target plate, followed by drying at ambient temperature. The dried sample was overlaid with $1 \mu$ of HCCA matrix solution, followed by air-drying at ambient temperature for crystallizing.

For the extended direct transfer method (EDT); a single colony was deposited directly on an assigned position of an MSP 96 target plate and was immediately overlaid with $1 \mu \mathrm{l}$ of $70 \%$ formic acid, then dried at ambient temperature. The sample was overlaid with $1 \mu \mathrm{l}$ of HCCA matrix solution, followed by air-drying at ambient temperature for crystallizing.

For the full extraction method (EX); a loopful of a colony of each bacterium scraped from the agar plate was suspended in $300 \mu$ of sterile distilled water in a micro-centrifuge tube, followed by the addition of $900 \mu \mathrm{l}$ ethanol and vortexing. The bacterial suspension was centrifuged at $16,000 \times g$ for $10 \mathrm{~min}$ to remove the supernatant.
The pellet was dried at ambient temperature and then resuspended in a mixture of $25 \mu \mathrm{l}$ of $70 \%$ formic acid and $25 \mu \mathrm{l}$ of acetonitrile by vortexing. After centrifugation at $16,000 \times g$ for $10 \mathrm{~min}$ to discard the pellet, the supernatant was carefully transferred into a new tube. One microliter of the resulting supernatant was deposited on an assigned position of an MSP 96 target plate, then dried at ambient temperature. The sample was overlaid with $1 \mu \mathrm{l}$ of HCCA matrix solution, followed by airdrying at ambient temperature for crystallizing. The MSP 96 target plate with prepared samples was immediately applied to MALDI-TOF MS.

\section{MALDI-TOF MS Analysis}

Bacterial identification of Staphylococcus strains was performed by the MALDI-TOF MS Microflex LT bench-top mass spectrometer (Bruker Daltonics). The measurements on the MALDI-TOF MS were performed using FlexControl software (version 3.0) within a mass range of 2,000 to 20,000 Da following calibration with a bacterial test standard (Bruker Daltonics). The generated mass spectrum of each sample was compared to a reference library in the MALDI biotyper database containing 5,627 reference spectra. The software calculated integrated pattern-matching algorithms and spectra were recorded as logarithms between 0 and 3.0. As specified by the manufacturer's instructions, $\log$ scores $\geq 2.0$ were accepted for identification at the species level and log scores of $<2.0$ and $\geq 1.7$ were taken as identification at the genus level or presumptive species level identification. Log scores below 1.7 were considered unreliable. Principal component analysis (PCA) and an MSP-based dendrogram using Biotyper software (Bruker Daltonics) were also performed to visualize intra-species similarities or variations between the Staphylococcus species and strains used in this study.

\section{$16 \mathrm{~S}$ rRNA Gene Sequencing}

The Staphylococcus isolates were cultured in nutrient broth (BD) and their DNAs were extracted using the G-Spin Bacterial Genomic DNA Extraction kit (Intron Biotechnology, Seongnam, Korea) according to the manufacturer's instructions. The $16 \mathrm{~S}$ rRNA gene of the isolate was amplified using 16S rRNA universal primer pairs (27F and 1492R) and the PCR products were purified using the QIAquick PCR Purification Kit (Qiagen, Hilden, Germany). Purified PCR products were sequenced and identified by comparison with the sequence of the National Biotechnology Information Center (NCBI) using the Basic Local Alignment Search Tool (BLAST) (Bionics, Korea).

\section{Results}

\section{MALDI-TOF MS Analysis of Staphylococcus Reference Strains}

The diagnostic ability of MALDI-TOF MS was evaluated with 19 reference strains by incubation period (Day-1, Day-2, 
Table 2. Performance of MALDI-TOF MS for the identification of Staphylococcus reference strains at genus level (log scores $\geq 1.7$ ) by sample preparation method and incubation period of bacteria culture.

\begin{tabular}{|c|c|c|c|c|c|c|c|c|c|}
\hline \multirow{2}{*}{ Strain } & \multicolumn{3}{|c|}{ Day 1} & \multicolumn{3}{|c|}{ Day 2} & \multicolumn{3}{|c|}{ Day 3} \\
\hline & $\mathrm{DT}^{\mathrm{a}}$ & EDT & EX & DT & EDT & EX & DT & EDT & EX \\
\hline S. aureus $(n=4)$ & $4(100 \%)$ & $4(100 \%)$ & $4(100 \%)$ & $4(100 \%)$ & $4(100 \%)$ & $4(100 \%)$ & $4(100 \%)$ & $4(100 \%)$ & $4(100 \%)$ \\
\hline S. epidermidis $(n=3)$ & $3(100 \%)$ & $3(100 \%)$ & $3(100 \%)$ & $3(100 \%)$ & $3(100 \%)$ & $3(100 \%)$ & $3(100 \%)$ & $3(100 \%)$ & $3(100 \%)$ \\
\hline S. capitis $(n=1)$ & $1(100 \%)$ & $1(100 \%)$ & $1(100 \%)$ & $1(100 \%)$ & $1(100 \%)$ & $1(100 \%)$ & $1(100 \%)$ & $1(100 \%)$ & $1(100 \%)$ \\
\hline S. caprae $(n=1)$ & $1(100 \%)$ & $1(100 \%)$ & $1(100 \%)$ & $1(100 \%)$ & $1(100 \%)$ & $1(100 \%)$ & $1(100 \%)$ & $1(100 \%)$ & $1(100 \%)$ \\
\hline S. haemolyticus $(n=1)$ & $1(100 \%)$ & $1(100 \%)$ & $1(100 \%)$ & $1(100 \%)$ & $1(100 \%)$ & $1(100 \%)$ & $1(100 \%)$ & $1(100 \%)$ & $1(100 \%)$ \\
\hline S. hominis $(n=1)$ & $1(100 \%)$ & $1(100 \%)$ & $1(100 \%)$ & $1(100 \%)$ & $1(100 \%)$ & $1(100 \%)$ & $1(100 \%)$ & $1(100 \%)$ & $1(100 \%)$ \\
\hline S. schleiferi $(n=1)$ & $1(100 \%)$ & $1(100 \%)$ & $1(100 \%)$ & $1(100 \%)$ & $1(100 \%)$ & $1(100 \%)$ & $0(0 \%)$ & $1(100 \%)$ & $1(100 \%)$ \\
\hline S. lugdunensis $(n=1)$ & $1(100 \%)$ & $1(100 \%)$ & $1(100 \%)$ & $1(100 \%)$ & $1(100 \%)$ & $1(100 \%)$ & $1(100 \%)$ & $1(100 \%)$ & $1(100 \%)$ \\
\hline S. saprophyticus $(n=1)$ & $1(100 \%)$ & $1(100 \%)$ & $1(100 \%)$ & $1(100 \%)$ & $1(100 \%)$ & $1(100 \%)$ & $1(100 \%)$ & $1(100 \%)$ & $1(100 \%)$ \\
\hline S. $x y l o s u s ~(n=1)$ & $1(100 \%)$ & $1(100 \%)$ & $1(100 \%)$ & $1(100 \%)$ & $1(100 \%)$ & $1(100 \%)$ & $1(100 \%)$ & $1(100 \%)$ & $1(100 \%)$ \\
\hline S. lentus $(n=1)$ & $1(100 \%)$ & $1(100 \%)$ & $1(100 \%)$ & $0(0 \%)$ & $0(0 \%)$ & $1(100 \%)$ & $0(0 \%)$ & $1(100 \%)$ & $1(100 \%)$ \\
\hline S. sciuri $(n=1)$ & $1(100 \%)$ & $1(100 \%)$ & $1(100 \%)$ & $1(100 \%)$ & $1(100 \%)$ & $1(100 \%)$ & $1(100 \%)$ & $1(100 \%)$ & $1(100 \%)$ \\
\hline S. warneri $(n=1)$ & $1(100 \%)$ & $1(100 \%)$ & $1(100 \%)$ & $1(100 \%)$ & $1(100 \%)$ & $1(100 \%)$ & $1(100 \%)$ & $1(100 \%)$ & $1(100 \%)$ \\
\hline S. pettenkoferi $(n=1)$ & $0(0 \%)$ & $1(100 \%)$ & $1(100 \%)$ & $1(100 \%)$ & $1(100 \%)$ & $1(100 \%)$ & $1(100 \%)$ & $1(100 \%)$ & $1(100 \%)$ \\
\hline Total $(n=19)$ & $18(94.7 \%)$ & $19(100 \%)$ & $19(100 \%)$ & $18(94.7 \%)$ & $18(94.7 \%)$ & $19(100 \%)$ & $17(89.5 \%)$ & $19(100 \%)$ & $19(100 \%)$ \\
\hline
\end{tabular}

${ }^{a} \mathrm{DT}$, direct transfer method; EDT, extended direct transfer method; EX, extraction method.

Table 3. Performance of MALDI-TOF MS for the identification of Staphylococcus reference strains at species level (log scores $\geq 2.0$ ) by sample preparation method and incubation period of bacteria culture.

\begin{tabular}{|c|c|c|c|c|c|c|c|c|c|}
\hline \multirow{2}{*}{ Strain } & \multicolumn{3}{|c|}{ Day 1} & \multicolumn{3}{|c|}{ Day 2} & \multicolumn{3}{|c|}{ Day 3} \\
\hline & DT & EDT & EX & DT & EDT & EX & DT & EDT & EX \\
\hline S. aureus $(n=4)$ & $4(100 \%)$ & $4(100 \%)$ & $4(100 \%)$ & $4(100 \%)$ & $4(100 \%)$ & $4(100 \%)$ & $4(100 \%)$ & $4(100 \%)$ & $4(100 \%)$ \\
\hline S. epidermidis $(n=3)$ & $2(66.7 \%)$ & $3(100 \%)$ & $3(100 \%)$ & $1(33.3 \%)$ & $2(66.7 \%)$ & $2(66.7 \%)$ & $0(0 \%)$ & $3(100 \%)$ & $1(33.3 \%)$ \\
\hline S. capitis $(n=1)$ & $0(0 \%)$ & $1(100 \%)$ & $1(100 \%)$ & $1(100 \%)$ & $1(100 \%)$ & $1(100 \%)$ & $1(100 \%)$ & $1(100 \%)$ & $1(100 \%)$ \\
\hline S. caprae $(n=1)$ & $0(0 \%)$ & $0(0 \%)$ & $1(100 \%)$ & $1(100 \%)$ & $1(100 \%)$ & $1(100 \%)$ & $0(0 \%)$ & $1(100 \%)$ & $1(100 \%)$ \\
\hline S. haemolyticus $(n=1)$ & $0(0 \%)$ & $1(100 \%)$ & $1(100 \%)$ & $0(0 \%)$ & $1(100 \%)$ & $1(100 \%)$ & $1(100 \%)$ & $1(100 \%)$ & $1(100 \%)$ \\
\hline S. hominis $(n=1)$ & $0(0 \%)$ & $1(100 \%)$ & $1(100 \%)$ & $0(0 \%)$ & $1(100 \%)$ & $1(100 \%)$ & $1(100 \%)$ & $1(100 \%)$ & $1(100 \%)$ \\
\hline S. schleiferi $(n=1)$ & $1(100 \%)$ & $1(100 \%)$ & $1(100 \%)$ & $1(100 \%)$ & $0(0 \%)$ & $1(100 \%)$ & $0(0 \%)$ & $1(100 \%)$ & $1(100 \%)$ \\
\hline S.lugdunensis $(n=1)$ & $1(100 \%)$ & $1(100 \%)$ & $1(100 \%)$ & $1(100 \%)$ & $1(100 \%)$ & $1(100 \%)$ & $1(100 \%)$ & $1(100 \%)$ & $1(100 \%)$ \\
\hline S. saprophyticus $(n=1)$ & $1(100 \%)$ & $1(100 \%)$ & $1(100 \%)$ & $1(100 \%)$ & $1(100 \%)$ & $1(100 \%)$ & $0(0 \%)$ & $1(100 \%)$ & $1(100 \%)$ \\
\hline S. $x y \operatorname{losus}(n=1)$ & $1(100 \%)$ & $1(100 \%)$ & $1(100 \%)$ & $1(100 \%)$ & $1(100 \%)$ & $1(100 \%)$ & $0(0 \%)$ & $1(100 \%)$ & $1(100 \%)$ \\
\hline S. lentus $(n=1)$ & $0(0 \%)$ & $0(0 \%)$ & $0(0 \%)$ & $0(0 \%)$ & $0(0 \%)$ & $0(0 \%)$ & $0(0 \%)$ & $0(0 \%)$ & $0(0 \%)$ \\
\hline S. sciuri $(n=1)$ & $1(100 \%)$ & $0(0 \%)$ & $0(0 \%)$ & $1(100 \%)$ & $1(100 \%)$ & $1(100 \%)$ & $1(100 \%)$ & $1(100 \%)$ & $0(0 \%)$ \\
\hline S. warneri $(n=1)$ & $1(100 \%)$ & $0(0 \%)$ & $1(100 \%)$ & $0(0 \%)$ & $0(0 \%)$ & $0(0 \%)$ & $0(0 \%)$ & $0(0 \%)$ & $1(100 \%)$ \\
\hline S. pettenkoferi $(n=1)$ & $0(0 \%)$ & $1(100 \%)$ & $1(100 \%)$ & $0(0 \%)$ & $1(100 \%)$ & $0(0 \%)$ & $1(100 \%)$ & $1(100 \%)$ & $0(0 \%)$ \\
\hline Total $(n=19)$ & $12(63.2 \%)$ & $15(78.9 \%)$ & $17(89.5 \%)$ & $12(63.2 \%)$ & $15(78.9 \%)$ & $15(78.9 \%)$ & $10(52.6 \%)$ & $17(89.5 \%)$ & $14(73.7 \%)$ \\
\hline
\end{tabular}

and Day-3) and by sample preparation method (DT, EDT, and EX) as shown in Tables 2 and 3. Overall diagnostic accuracy considering identification at the genus and species levels revealed that almost 19 reference strains were identified correctly. At the genus identification level, the EDT and EX sample preparation methods provided excellent identification results with almost $100 \%$ accuracy regardless of incubation period. Meanwhile, the DT method 
showed relatively low identification accuracy (Table 2). At the species identification level, the EX method at Day-1 revealed the best diagnostic ability ( $89 \%$ accuracy) (Table 3). The EDT method revealed a constant identification yield (between $78 \%$ to $89 \%$ accuracy) regardless of incubation period, whereas the DT method yielded low accuracy (less than $64 \%$ accuracy) with discrepant results. The relatively low accuracy seen when using the DT method may be due to the absence of formic acid treatment and the simple steps in the DT method that provide relatively low protein extraction from bacterial cell walls. Based on our comparison of these three preparation methods, the EX extraction method was selected for identification of Staphylococcus isolates.

The analyzed spectra obtained by MALDI-TOF MS and the MSP-based dendrogram for the 19 reference strains yielded by the EX method at Day-1, are shown in Figs. 1 and 2. The MALDI-TOF MS analysis yielded clearly different spectra. Their overall dendrogram (see Fig. 2) reveals clean distinctions between Staphylococcus species. Staphylococcus strains belonging to the same species (4 strains of $S$. aureus and 3 strains of $S$. epidermidis) were clustered together apart from other Staphylococcus species and the dendrogram between the S. aureus or S. epidermidis strains suggested the potential for further detailed discrimination within strains by species. The clustered groups of Staphylococcus species were not in accord with the 16S rRNA gene sequencing dendrogram reported previously [22]. Interestingly, the dendrogram suggested clean discrimination between $S$. capitis and S. caprae, and between $S$. saprophyticus and S. xylosus, while $16 \mathrm{~S}$ rRNA gene sequencing analysis did not distinguish between them $[6,22]$.

\section{MALDI-TOF MS Analysis of Staphylococcus Strains Isolated from Food Samples}

A total of 96 Staphylococcus strains were isolated from food samples including jeotgal (traditional Korean fermented seafood; three kinds of jeotgal used in this study were shrimp jeotgal, shellfish jeotgal, and fish jeotgal), salted Chinese cabbage, and raw milk (obtained from a local milk farm without pasteurization), purchased at a local market in South Korea. For identification of all Staphylococcus isolates, we used the EX method at Day-1. All 96 strains
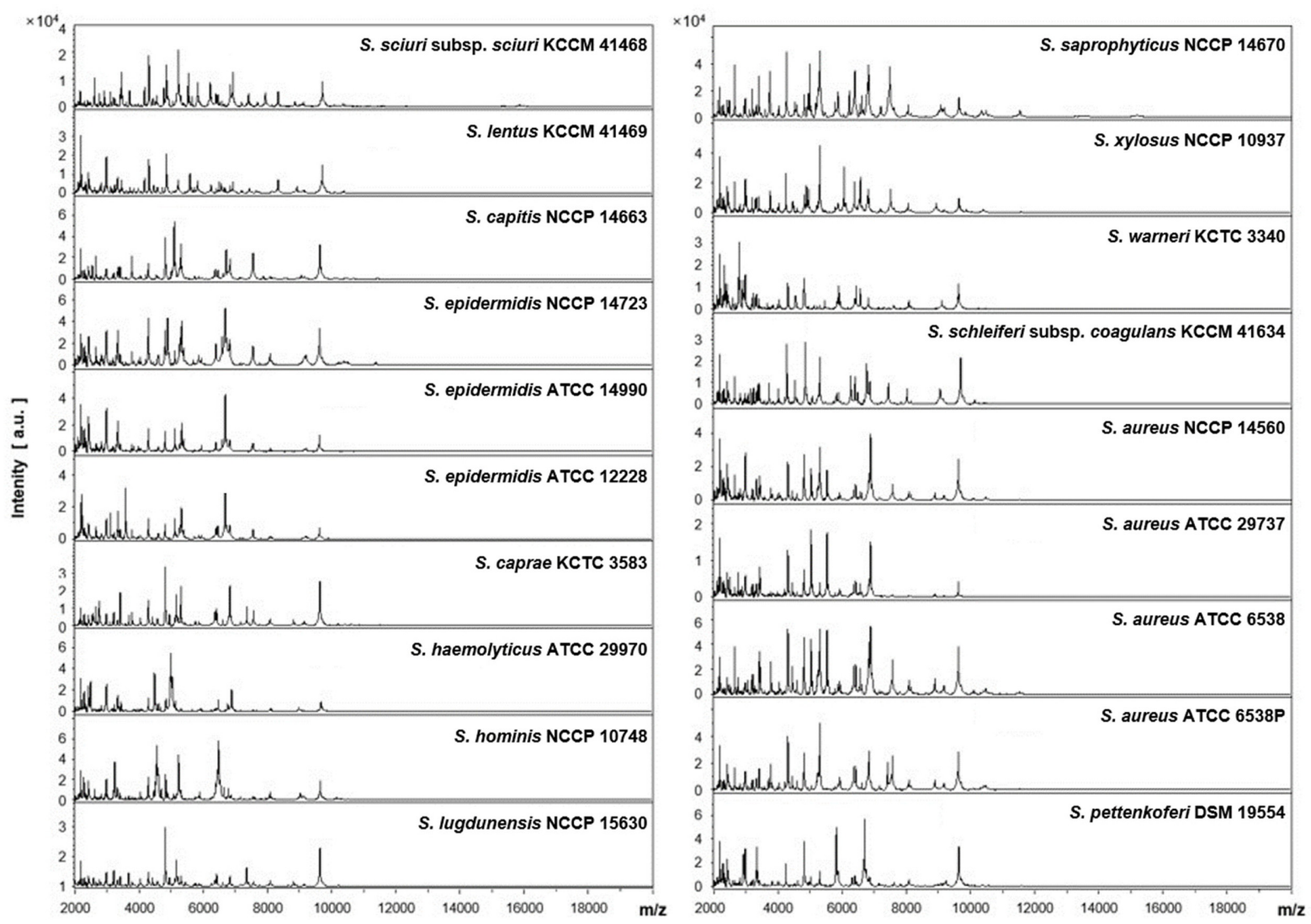

Fig. 1. MALDI-TOF MS spectra of 19 Staphylococcus reference strains in the 2,000 to 20,000 Da range. 


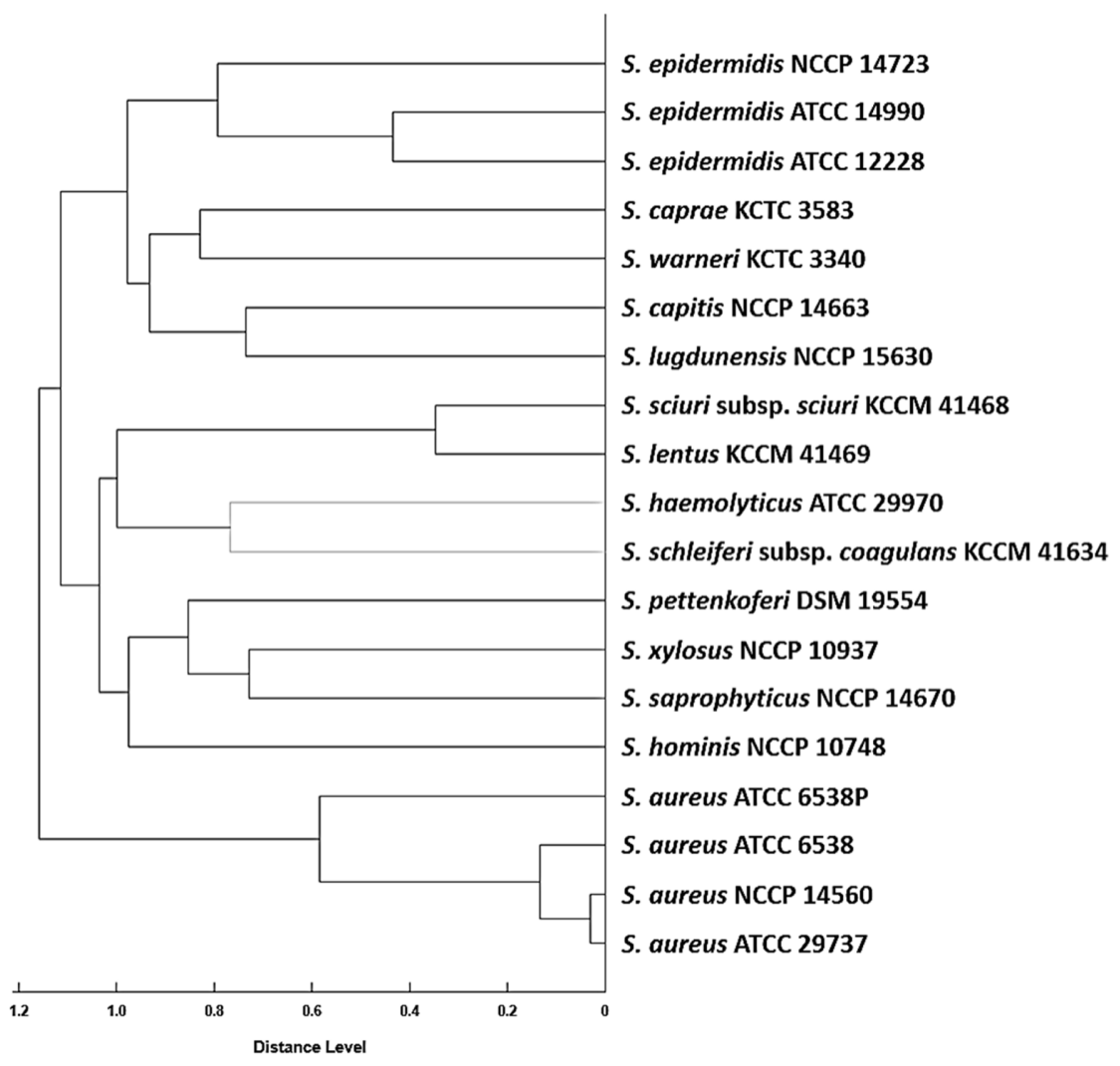

Fig. 2. Main spectra profile (MSP)-based dendrogram from MALDI-TOF MS spectra of the 19 Staphylococcus reference strains and species clustering of the isolates analyzed with the reference strains.

Distance values below the dendrogram are relative and normalized to a maximal value of 1,000.

were subjected to analysis by MALDI-TOF MS and acquired spectra were compared with the MALDI Biotyper database. The isolates were identified as Staphylococcus genus and consisted of 7 Staphylococcus species as shown in Table 4. Various Staphylococcus species were isolated from three kinds of jeotgal and S. epidermidis was the major species isolated from jeotgal foods. By contrast, the two other foods had only a single Staphylococcus species (raw milk: S. epidermidis; salted Chinese cabbage: S.hominis). Additionally, the ubiquitous presence of $S$. epidermidis in various foods was confirmed in this study. The results of the log score value obtained by MALDI-TOF MS are listed in Table 5. All Staphylococcus isolates (100\% accuracy) were correctly identified at the species or genus level, of which $78(81.3 \%$ accuracy) were identified at the species level and $18(18.8 \%$ accuracy) were identified at the genus level.

Table 4. Identified population of Staphylococcus species using MALDI-TOF MS analysis isolated from various foods in Korea.

\begin{tabular}{|c|c|c|c|c|c|c|c|c|}
\hline \multirow{2}{*}{ Food source } & \multicolumn{7}{|c|}{ Number of Staphylococcus species } & \multirow{2}{*}{ Tota } \\
\hline & S. capitis & S. epidermidis & S. equorum & S. hominis & S. Pasteuri & S. simulans & S. warneri & \\
\hline Shrimp jeotgal $^{\mathrm{a}}$ & 3 & 12 & 16 & 0 & 8 & 12 & 1 & 52 \\
\hline Shellfish jeotgal & 3 & 6 & 0 & 0 & 1 & 0 & 0 & 10 \\
\hline Fish jeotgal & 0 & 10 & 0 & 1 & 0 & 0 & 0 & 11 \\
\hline Salted Chinese cabbage ${ }^{b}$ & 0 & 0 & 0 & 9 & 0 & 0 & 0 & 9 \\
\hline Raw milk & 0 & 14 & 0 & 0 & 0 & 0 & 0 & 14 \\
\hline Total & 6 & 42 & 16 & 10 & 9 & 12 & 1 & 96 \\
\hline
\end{tabular}

a Jeotgal is traditional Korean fermented seafood (shrimp jeotgal, shellfish jeotgal, fish jeotgal).

${ }^{\mathrm{b}}$ Salted Chinese cabbage is a major ingredient of kimchi, a traditional Korean fermented food. 
Table 5. Identification of 96 Staphylococcus isolates by MALDITOF MS.

\begin{tabular}{lccc}
\hline \multirow{2}{*}{$\begin{array}{c}\text { Species } \\
\text { (no. of isolates) }\end{array}$} & \multicolumn{3}{c}{ No. of Staphylococcus species with results ${ }^{\mathrm{a}}$. } \\
\cline { 2 - 4 } S. 2.000 & $1.700-1.999$ & $\leq 1.699$ \\
\hline S. epidermidis (42) & 40 & 2 & 0 \\
S. pasteuri (9) & 9 & 0 & 0 \\
S. capitis (6) & 6 & 0 & 0 \\
S. hominis (10) & 7 & 3 & 0 \\
S. equorum (16) & 4 & 12 & 0 \\
S. simulans (12) & 11 & 1 & 0 \\
S. warneri (1) & 1 & 0 & 0 \\
Total isolates (96) & 78 & 18 & 0 \\
\hline
\end{tabular}

${ }^{a} \geq 2.000$ : species-level-identification; 1.700 1.999: genus-level-identification; $\leq$ 1.699: not reliable identification.

\section{PCA and MSP-Based Dendrogram of Isolated Staphylococcus}

PCA using Biotyper software was accomplished to identify intra-species similarity and variation between the 96 isolated Staphylococcus strains and two-dimensional plots were obtained as shown in Fig. 3. All 96 strains of Staphylococcus species were separated into 6 groups (Group 1:

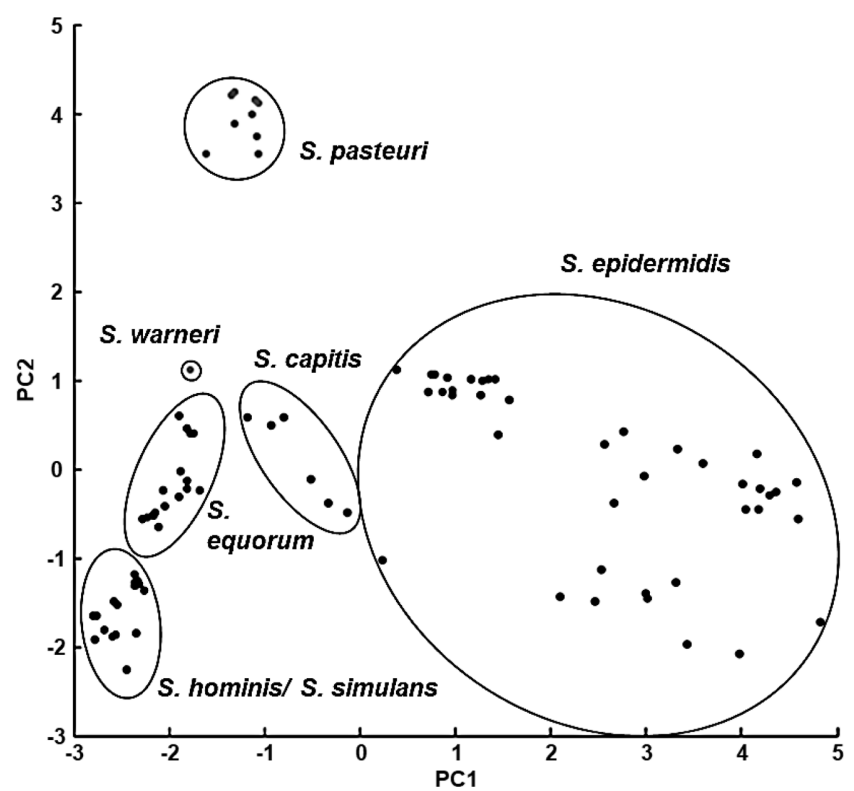

Fig. 3. Two dimensional plots of the spectra of 96 Staphylococcus isolates generated by principal component analysis (PCA).

The isolates are clearly separated into 6 groups, which visually demonstrates the heterogeneity of the protein spectra among Staphylococcus species. Each dot indicates the spectrum of one isolate.
S. pasteuri; Group 2: S. warneri; Group 3: S. epidermidis; Group 4: S. equorum; Group 5: S. capitis; Group 6: S. simulans and S. hominis) based on PCA. In contrast to previously reported groups of Staphylococcus species based on 16S rRNA gene sequencing [21], the MALDI-TOF MS method used in this study revealed novel clustering between 7 species of Staphylococcus isolates. S. capitis $(n=6)$ was separated from $S$. epidermidis $(n=42)$. S. equorum $(n=16)$, S. simulans $(n=12), S$. warneri $(n=1), S$. pasteuri $(n=9)$, and $S$. hominis $(n=10)$ were clustered into one group, which were divided into different groups previously [21] but subdivided into S. hominis / S. simulans on two-dimensional plots. The MSP dendrogram of 96 isolates based on the

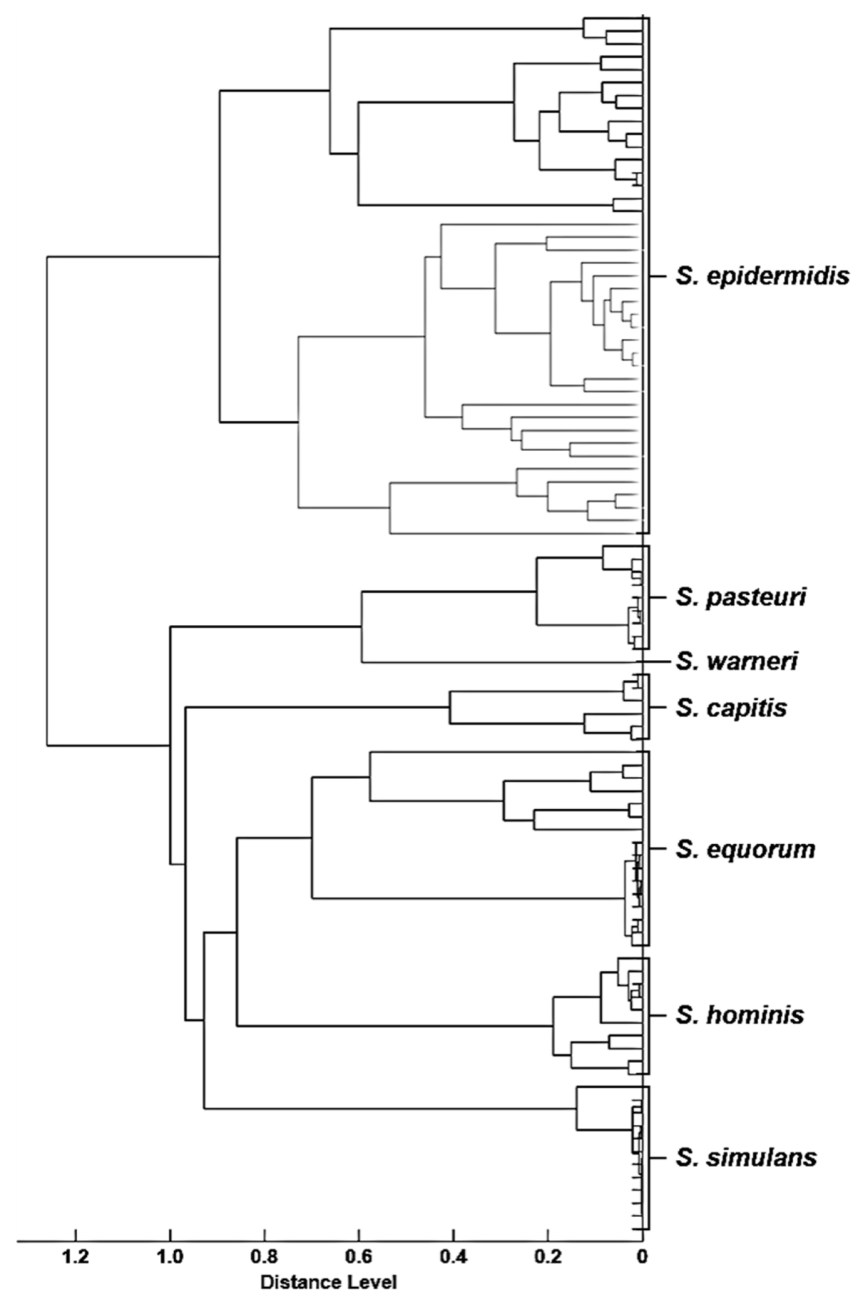

Fig. 4. Main spectra profile (MSP)-based dendrogram from MALDI-TOF MS spectra of the 96 Staphylococcus strains isolated from various foods.

Clusters are based on the Staphylococcus species level. Distance values below the dendrogram are relative and normalized to a maximal value of 1,000 . 
Table 6. Comparison of the identification results of Staphylococcus isolates by $16 \mathrm{~S}$ rRNA gene sequencing and MALDI-TOF MS.

\begin{tabular}{|c|c|c|c|}
\hline Origin & MALDI-TOF MS results $\left(n^{a}\right)$ & 16S rRNA gene sequencing results (NCBI accession no.) & Identity \\
\hline \multirow[t]{6}{*}{ Shrimp jeotgal } & S. capitis (3) & S. capitis (MK318575.1) & $100 \%$ \\
\hline & S. epidermidis (12) & S. epidermidis (KY753228.1) & $100 \%$ \\
\hline & S. equorum (16) & S. equorum (MK253324.1), S. haemolyticus (MF578766.1) & $100 \%$ \\
\hline & S. pasteuri (8) & S. pasteuri (KT036409.1), S. warneri (KT720133.1) & $100 \%$ \\
\hline & S. simulans (12) & S. simulans(KC849422.1) & $100 \%$ \\
\hline & S. warneri (1) & S. warneri (KT153529.1), S. pasteuri (KT427912.1) & $100 \%$ \\
\hline \multirow[t]{3}{*}{ Shellfish jeotgal } & S. capitis (3) & S. capitis (KT027728.1) & $100 \%$ \\
\hline & S. epidermidis (6) & S. epidermidis (MG645276.1) & $100 \%$ \\
\hline & S. pasteuri (1) & S. pasteuri (MH158278.1), S. warneri (MG920271.1) & $100 \%$ \\
\hline \multirow[t]{2}{*}{ Fish jeotgal } & S. epidermidis (10) & S. epidermidis (KT427443.1) & $100 \%$ \\
\hline & S. hominis (1) & S. hominis (MH715220.1) & $100 \%$ \\
\hline Salted Chinese cabbage & S. hominis (9) & S. hominis (MK318620.1) & $100 \%$ \\
\hline Raw milk & S. epidermidis (14) & S. epidermidis (MH118521.1) & $100 \%$ \\
\hline
\end{tabular}

${ }^{\mathrm{a}}$ Number of isolates.

PCR clustering results revealed more detailed relationships between Staphylococcus strains at the species level as shown in Fig. 4. The dendrogram primarily revealed that each species derived from Staphylococcus isolates was clearly clustered.

\section{S rRNA Gene Sequencing}

The identification results of comparing $16 \mathrm{~S}$ rRNA gene sequencing and MALDI-TOF MS are listed in Table 6. Based on the 16S rRNA gene sequencing, 6 isolates were identified as S. capitis (NCBI Accession No. NK318575.1 and KT027728.1), and 12 isolates were identified as S. simulans (KC849422.1), and 10 isolates were identified as S. hominis (MH715220.1 and MK318620.1), and 42 isolates were identified as S. epidermidis (MH118521.1, MG6452761.1, KT427443.1, and KY753228.1). The remaining isolates were identified as S. equorum (MK253324.1) or S. haemolyticus (MF578766.1) and S. pasteuri (MH158278.1, KT036409.1, and KT427912.1) or S. warneri (MG920271.1, KT720133.1, and KT153529.1), respectively.

\section{Discussion}

A phylogenetic study of 38 species in the Staphylococcus genus was previously reported based on the sequence alignment of $16 \mathrm{~S}$ rRNA genes [21]. 16S rRNA gene sequencing analysis was unable to distinguish between some Staphylococcus species (e.g. among S. saccharolyticus, S. capitis subsp. urealyticus and S. caprae, and between two subspecies of S. cohnii). Palys et al. described 16S rRNA gene sequencing as having relatively limited or moderate identification ability between closely related bacterial populations due to limitations in its discriminatory power [23]. In the present study, MALDI-TOF MS analysis showed a reliable ability to distinguish between closely related Staphylococcus species of reference strains as shown in Fig. 2 (between S. capitis, S. caprae and S. epidermidis; between S. hominis, S. heamolyticus and S. lugdunensis; between $S$. saprophyticus and S. xylosus; and between S. sciuri and S. lentus). Additionally, the results shown in Table 2 support the ability of MALDI-TOF MS to identify Staphylococcus at the species level. Therefore, MALDI-TOF MS analysis could be a reliable diagnostic method and be expected to serve as a counterpart method against $16 \mathrm{~S}$ rRNA gene analysis in classification of bacteria.

In order to accurately identify Staphylococcus species using MALDI-TOF MS, sample preparation methods should be established. In previous reports, the impact of the protein extraction method and incubation period were compared on the yield of MALDI-TOF MS for identification in Clostridium spp. and gram-positive cocci [21, 24]. Chean et al. found that both EDT and EX sample preparations showed similar performance of over $96 \%$ to $100 \%$ accuracy at the species level of isolated Clostridium regardless of incubation period [3]. Also, Schulthess et al. found that overall, both protein extraction methods performed similar identification yields with isolated strains of gram-positive cocci [21]. These studies revealed that the EX sample 
preparation method performed at higher or similar yield compared with the EDT method. Meanwhile, incubation period did not seem to be a critical factor in either the EDT or EX methods for the performance of MALDI-TOF MS even at the species level of bacterial identification, in contrast to the DT method.

We evaluated the performance of MALDI-TOF MS for the identification of Staphylococcus by sample preparation method efficacy (DT, EDT, EX) and incubation period (Day-1, Day-2, Day-3) as shown in Table 2. Overall results revealed the EX method had the highest and most stable performance at the species level in Staphylococcus reference strains, similar to the results previously reported for Clostridium spp. and gram-positive cocci [21, 24]. Pretreatment sample preparation using the EX protocol improved the mass spectral log score by reducing background signals and generating sufficient protein signals. Treated sample spectra also were better correlated with the Biotyper database as its spectra were created by EX sample preparation [10]. We also investigated how incubation period affected the improvement in identification rate and showed that $24 \mathrm{~h}$ yielded the most successful identification rate. This culture condition may generate sufficient biomass for MALDI-TOF analysis. The dendrogram created from Staphylococcus isolates spectra showed two major clusters (see Fig. 4). The PCA procedure separated all Staphylococcus species into 6 distinctive clusters, which demonstrates that the MALDI-TOF MS method used in this study was reliable for Staphylococcus identification.

We compared identification results of Staphylococcus isolates by $16 \mathrm{~S}$ rRNA gene sequencing and MALDI-TOF MS. S. simulans, S. hominis, and S. epidermidis were identified as one species in both methods. However, some $16 \mathrm{~S}$ rRNA gene sequencing results revealed two candidate Staphylococcus species instead of providing one specific species, whereas MALDI-TOF MS clearly identified those strains as S. equorum, S. pasteuri, and S. warneri. These results are consistent with previous studies that Staphylococcus species cannot be accurately distinguished using the $16 \mathrm{~S}$ rRNA gene sequence [25]. Our results conclude that MALDI-TOF MS can be used to accurately identify Staphylococcus to the species level. In order to identify Staphylococcus isolates by $16 \mathrm{~S}$ rRNA gene sequencing, a lot of time is consumed in culturing, DNA extraction, and sequencing. However, for MALDI-TOF, one target plate (96 isolates) after the strain culture can be identified by the extraction method within 3 hours [26].

The identified Staphylococcus species were compared to investigate bacterial diversity in various food habitats. The populations of CoNS in various types of foodstuffs including cheese, cured meat, sausage, smoked fish, fermented foods, and starter cultures in Europe have been reported in previous studies (e.g. S. xylosus, S. epidermidis, S. lentus, S. saprophyticus, S. hyicus, S. simulans, S. carnosus, S. condimenti, S. equorum, S. piscifermentans, S. succinus) [2, 5]. In the present study, Staphylococcus strains were isolated from various foods including traditional fermented foods and were identified as the seven species shown in Table 4. The dominant Staphylococcus species in salted Chinese cabbage and raw milk seemed unique and specific to each food matrix, while the populations of Staphylococcus species in jeotgal foods were diverse and revealed a different set of species compared to that found in European foods.

In this study, the identification of isolated Staphylococcus species from various foods was performed using MALDITOF MS. Additionally, an MSP-based dendrogram and the PCA procedure enabled further discrimination between Staphylococcus strains at the species level. This study is a good example of subtyping Staphylococcus at the strain level and provides valuable information for practical and extended application of MALDI-TOF MS for food monitoring and epidemiological study.

\section{Acknowledgments}

This work was funded by the Strategic Initiative for Microbiomes in Agriculture and Food, Ministry of Agriculture, Food and Rural Affairs, Republic of Korea (Grant number 918005-4).

\section{Conflict of Interest}

The authors have no financial conflicts of interest to declare.

\section{References}

1. Becker K, Heilmann C, Peters G. 2014. Coagulase-negative staphylococci. Clin. Microbiol. Rev. 27: 870-926.

2. Zell C, Resch M, Rosenstein R, Albrecht T, Hertel C, Götz F. 2008. Characterization of toxin production of coagulasenegative staphylococci isolated from food and starter cultures. Int. J. Food Microbiol. 127: 246-251.

3. Podkowik M, Park JY, Seo KS, Bystroń J, Bania J. 2013. Enterotoxigenic potential of coagulase-negative staphylococci. Int. J. Food Microbiol. 163: 34-40.

4. Irlinger F. 2008. Safety assessment of dairy microorganisms: coagulase-negative staphylococci. Int. J. Food Microbiol. 126: 302-310. 
5. Chajęcka-Wierzchowska W, Zadernowska A, Nalepa B, Sierpinska M, Łaniewska-Trokenheim Ł. 2015. Coagulasenegative staphylococci (CoNS) isolated from ready-to-eat food of animal origin - phenotypic and genotypic antibiotic resistance. Food Microbiol. 46: 222-226.

6. Ayeni FA, Andersen C, Nørskov-Lauritsen N. 2017. Comparison of growth on mannitol salt agar, matrix-assisted laser desorption/ionization time-of-flight mass spectrometry, VITEK 2 with partial sequencing of 16S rRNA gene for identification of coagulase-negative staphylococci. Microb. Pathog. 105: 255-259.

7. Hwang SM, Kim MS, Park KU, Song J, Kim EC. 2011. Tuf gene sequence analysis has greater discriminatory power than $16 \mathrm{~S}$ rRNA sequence analysis in identification of clinical isolates of coagulase-negative staphylococci. J. Clin. Microbiol. 49: 4142-4149.

8. Bergeron M, Dauwalder O, Gouy M, Freydiere AM, Bes M, Meugnier H, et al. 2011. Species identification of staphylococci by amplification and sequencing of the tuf gene compared to the gap gene and by matrix-assisted laser desorption ionization time-of-flight mass spectrometry. Eur. J. Clin. Microbiol. Infect. Dis. 30: 343-354.

9. Drancourt M1, Raoult D. 2002. rpoB gene sequence-based identification of Staphylococcus species. J. Clin. Microbiol. 40: 1333-1338.

10. Rahi P, Prakash O, Shouche YS. 2016. Matrix-assisted laser desorption/ionization time-of-flight mass-spectrometry (MALDI-TOF MS) based microbial identifications: challenges and scopes for microbial ecologists. Front. Microbiol. 30: 1359.

11. Cho Y, Kim E, Lee Y, Han SK, Choo DW, Kim YR, et al. 2017. Rapid and accurate identification of Pediococcus species isolated from Korean fermented foods by MALDI-TOF MS with local database extension. Int. J. Syst. Evol. Microbiol. 67: 744-752.

12. Kim E, Cho Y, Lee Y, Han SK, Kim CG, Choo DW, et al. 2017. A proteomic approach for rapid identification of Weissella species isolated from Korean fermented foods on MALDI-TOF MS supplemented with an in-house database. Int. J. Food Microbiol. 243: 9-15.

13. Ahmadsah LSF, Kim E, Jung YS, Kim HY. 2018. Identification by PCR-DGGE, SDS-PAGE, and MALDI-TOF MS of LAB and fungi in laru, a fermented starter. J. Microbiol. Biotechnol. 28: 32-39.

14. Lee Y, Cho Y, Kim E, Kim HJ, Kim HY. 2018. Identification of lactic acid bacteria in Galchi- and Myeolchi-jeotgal by $16 \mathrm{~S}$ rRNA gene sequencing, MALDI-TOF mass spectrometry, and PCR-DGGE. J. Microbiol. Biotechnol. 28: 1112-1121.

15. Cameron M, Barkema HW, De Buck J, De Vliegher S, Chaffer M, Lewis J, et al. 2017. Identification of bovineassociated coagulase-negative staphylococci by matrix-assisted laser desorption/ionization time-of-flight mass spectrometry using a direct transfer protocol. J. Dairy Sci. 100: 2137-2147.
16. Zhu W, Sieradzki K, Albrecht V, McAllister S, Lin W, Stuchlik O, et al. 2015. Evaluation of the Biotyper MALDITOF MS system for identification of Staphylococcus species. J. Microbiol. Methods 117: 14-17.

17. Freiwald A, Sauer S. 2009. Phylogenetic classification and identification of bacteria by mass spectrometry. Nature Protoc. 4: 732-742.

18. Cho Y, Kim E, Han SK, Yang SM, Kim MJ, Kim HJ, et al. 2017. Rapid identification of Vibrio species isolated from the southern coastal regions of Korea by MALDI-TOF mass spectrometry and comparison of MALDI sample preparation methods. J. Microbiol. Biotechnol. 27: 1593-1601.

19. Ruaro A, Andrighetto C, Torriani S, Lombardi A. 2013. Biodiversity and characterization of indigenous coagulasenegative staphylococci isolated from raw milk and cheese of North Italy. Food Microbiol. 34: 106-111.

20. Haigh J, Degun A, Eydmann M, Millar M, Wilks M. 2011. Improved performance of bacterium and yeast identification by a commercial matrix-assisted laser desorption ionizationtime of flight mass spectrometry system in the clinical microbiology laboratory. J. Clin. Microbiol. 49: 3441.

21. Schulthess B, Brodner K, Bloemberg GV, Zbinden R, Böttger EC, Hombach M. 2013. Identification of gram-positive cocci by use of matrix-assisted laser desorption ionization-time of flight mass spectrometry: comparison of different preparation methods and implementation of a practical algorithm for routine diagnostics. J. Clin. Microbiol. 51: 1834-1840.

22. Takahashi T, Satoh I, Kikuchi N. 1999. Phylogenetic relationships of 38 taxa of the genus Staphylococcus based on 16S rRNA gene sequence analysis. Int. J. Syst. Bacteriol. 49: 725-728.

23. Palys T, Nakamura LK, Cohan FM. 1997. Discovery and classification of ecological diversity in the bacterial world: the role of DNA sequence data. Int. J. Syst. Bacteriol. 47: 1145-1156.

24. Chean R, Kotsanas D, Francis MJ, Palombo EA, Jadhav SR, Awad MM, et al. 2014. Comparing the identification of Clostridium spp. by two matrix-assisted laser desorption ionization-time of flight (MALDI-TOF) mass spectrometry platforms to $16 \mathrm{~S}$ rRNA PCR sequencing as a reference standard: a detailed analysis of age of culture and sample preparation. Anaerobe 30: 85-89.

25. Ghebremedhin B, Layer F, König W, König B. 2008. Genetic classification and distinguishing of Staphylococcus species based on different partial gap, 16S rRNA, hsp60, rpoB, sodA, and tuf gene sequences. J. Clin. Microbiol. 46: 1019-1025.

26. Eigner U, Holfelder M, Oberdorfer K, Betz-Wild U, Bertsch D, Fahr AM. 2009. Performance of a matrix-assisted laser desorption ionization-time-of-flight mass spectrometry system for the identification of bacterial isolates in the clinical routine laboratory. Clin. Lab. 55: 289-296. 